Evaluation of illumination system uniformity for wide-field biomedical hyperspectral imaging

This content has been downloaded from IOPscience. Please scroll down to see the full text.

2017 J. Opt. 19045301

(http://iopscience.iop.org/2040-8986/19/4/045301)

View the table of contents for this issue, or go to the journal homepage for more

Download details:

IP Address: 131.111.184.102

This content was downloaded on 10/05/2017 at 15:06

Please note that terms and conditions apply.

You may also be interested in:

Unconventional methods of imaging: computational microscopy and compact implementations

Euan McLeod and Aydogan Ozcan

Phytos: a portable goniometer for in situ spectro-directional measurements of leaves

Lapo Lolli, Marco Pisani, Mauro Rajteri et al.

Compound hyperbolic concentrator-type reflectors for liquid crystal display backlightsystems

Xingpeng Yang, Yingbai Yan, Di Feng et al.

Multimodal confocal hyperspectral imaging microscopy with wavelength sweeping source

Young-Duk Kim, Dukho Do, Hongki Yoo et al.

Fast processing spectral discrimination for hyperspectral imagers based on interferometry

M Zucco and M Pisani

Simulation of micro-optical systems including microlens arrays

Norbert Lindlein

Volumetric x-ray coherent scatter imaging of cancer in resected breast tissue: a Monte Carlo study using virtual anthropomorphic phantoms

Manu N Lakshmanan, Brian P Harrawood, Ehsan Samei et al.

Oxygenation and perfusion monitoring with a hyperspectral camera system for chemical based tissue analysis of skin and organs

Amadeus Holmer, Florian Tetschke, Jörg Marotz et al. 


\title{
Evaluation of illumination system uniformity for wide-field biomedical hyperspectral imaging
}

\author{
Travis W Sawyer, A Siri Luthman and Sarah E Bohndiek ${ }^{1}$ \\ Department of Physics and Cancer Research UK Cambridge Institute, University of Cambridge, \\ Cambridge, United Kingdom \\ E-mail: ts661@cam.ac.uk, asnl2@cam.ac.uk and seb53@cam.ac.uk
}

Received 25 October 2016, revised 10 February 2017

Accepted for publication 20 February 2017

Published 17 March 2017

\begin{abstract}
Hyperspectral imaging (HSI) systems collect both spatial (morphological) and spectral (chemical) information from a sample. HSI can provide sensitive analysis for biological and medical applications, for example, simultaneously measuring reflectance and fluorescence properties of a tissue, which together with structural information could improve early cancer detection and tumour characterisation. Illumination uniformity is a critical pre-condition for quantitative data extraction from an HSI system. Non-uniformity can cause glare, specular reflection and unwanted shading, which negatively impact statistical analysis procedures used to extract abundance of different chemical species. Here, we model and evaluate several illumination systems frequently used in wide-field biomedical imaging to test their potential for HSI. We use the software LightTools and FRED. The analysed systems include: a fibre ring light; a light emitting diode (LED) ring; and a diffuse scattering dome. Each system is characterised for spectral, spatial, and angular uniformity, as well as transfer efficiency. Furthermore, an approach to measure uniformity using the Kullback-Leibler divergence (KLD) is introduced. The KLD is generalisable to arbitrary illumination shapes, making it an attractive approach for characterising illumination distributions. Although the systems are quite comparable in their spatial and spectral uniformity, the most uniform angular distribution is achieved using a diffuse scattering dome, yielding a contrast of 0.503 and average deviation of 0.303 over a $\pm 60^{\circ}$ field of view with a $3.9 \%$ model error in the angular domain. Our results suggest that conventional illumination sources can be applied in HSI, but in the case of low light levels, bespoke illumination sources may offer improved performance.
\end{abstract}

Keywords: hyperspectral imaging, illumination, modelling, simulation, ray tracing

(Some figures may appear in colour only in the online journal)

\section{Introduction}

Hyperspectral imaging (HSI) is based on the acquisition of a three-dimensional data cube (figure 1), composed of two spatial dimensions $(x, y)$ and one spectral dimension $(\lambda)$. The hyperspectral data cube is constructed using one of four major acquisition modes: spatial-scanning [1], spectral-scanning [2], spatio-spectral scanning [3], and snapshot (non-scanning) [4].

\footnotetext{
1 Author to whom any correspondence should be addressed.
}

HSI is emerging as a new modality for biomedical imaging, with potential for wide ranging applications based on the combination of information available from both morphological and chemical features [2]. In particular, recent advances in HSI technology [5-7] have facilitated analysis of the reflectance and fluorescence characteristics of tissue, which may improve cancer diagnosis and monitoring of disease progression [2].

Underlying tissue pathology can be assessed using tissue optical properties by acquiring data with a wide-field HSI 


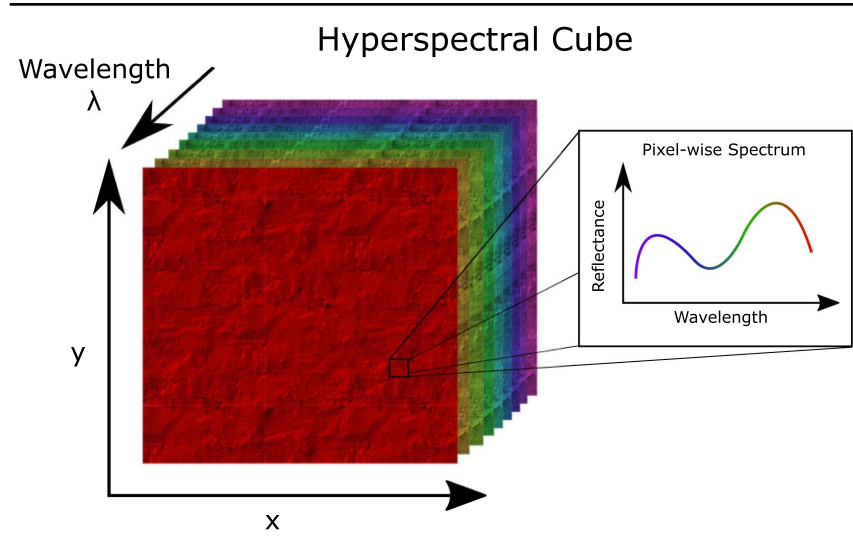

Figure 1. The hyperspectral cube is composed of two spatial dimensions $(x, y)$ and one spectral dimension $(\lambda)$. For each spatial pixel, a full spectrum is captured.

system applied to excised tissue samples. Both broadband illumination for diffuse reflectance [8,9] and narrowband illumination for fluorescence imaging $[10,11]$ have been used to date, demonstrating the feasibility of using HSI to study tissue pathology. To retrieve clinically relevant diagnostic information from wide-field HSI data, a given spectral signature must be consistently represented in all spatial parts of the image [5]. To achieve this, the whole sample must be equally well illuminated across the entire spectrum with high efficiency, without spatial (figure 2(A)), angular (figure 2(B)) or spectral non-uniformities (figure $2(\mathrm{C})$ ). This is true regardless of the data acquisition mode. When recording data from freeform geometries such as biological tissue, the illumination can be particularly sensitive to angular uniformity, where the non-flat nature of excised tissue samples can cast shadows and other occlusions. Preprocessing techniques such as normalisation have been used to minimise the impact of non-uniform sample illumination [13], however, such operations may distort the integrity of the final processed image data by introducing noise, resulting in an associated dynamic range penalty [14]. Low-quality data arising from illumination non-uniformity significantly reduces the performance of multivariate statistical analysis techniques [12], hence it is beneficial to optimise the illumination according to spatial, angular and spectral uniformity.

Common lighting solutions for wide-field imaging employ the concepts of merging irradiance distributions and scattering light. Such approaches effectively average a series of individual, potentially non-uniform, distributions to produce a more diffuse and homogeneous result [5]. Three of the most common examples of these techniques include fibre optic [12] or light emitting diode (LED) [15] ring lights, and dome illumination [16, 17]. While spatial non-uniformities can be measured by imaging a uniformly reflecting target, evaluating angular uniformity is experimentally more challenging, needing complicated and specialised equipment. Furthermore, while conventional (non-spectral) imaging systems integrate total irradiance across all wavelengths of light incident at the detector, HSI is sensitive to any spectral nonuniformity in the optical elements delivering the illumination or the light source (figures 2(C) and (D)).
Here, we use ray-tracing simulations, commonly used for optical design and analysis, to evaluate spatial, angular, and spectral illumination uniformity for three common illumination systems used in wide-field HSI. Previous work has experimentally investigated the spatial and angular uniformity of fibre optic ring lights and illumination domes [5]. We model both of these geometries, as well as an LED ring system. In addition to characterising uniformity in the spatial, angular, and spectral domains, we also consider the efficiency of each system. Uniformity is assessed using two standard metrics (contrast, average deviation) and a new generalised approach based on the Kullback-Leibler divergence (KLD) for each of spatial, angular and spectral uniformity [5, 18]. The KLD is an extension of the concept of entropy, which has previously been applied to characterise the uniformity of an illumination distribution, given perfectly uniform illumination has zero entropy [5]. The KLD is a measure of the difference between two probability distributions and has previously been proposed as a means to assess illumination levels, classifying brightness and contrast for imaging enhancement [19]; to the best of our knowledge, the assessment shown here is the first attempt to apply this concept as a uniformity metric. Based on our simulations, we find that the diffuse scattering dome provides the most uniform illumination solution for HSI.

\section{Methods}

\subsection{System models}

We modelled and evaluated three different illumination systems: a commercially available fibre ring light (FRL, figures 3(A) and (D); FRI61F50, Thorlabs, USA); a custommade LED ring (figures 3(B) and (E)); and a commercial diffuse dome illumination system (figures 3(C) and (F); MBDL306, Vision Light Tech, USA). To perform the comparison, each was modelled using the optical design and analysis software LightTools (Synopsis, Pasadena CA) and FRED (Photon Engineering, Tucson AZ).

2.1.1. Fibre ring light. The FRL is a professionally manufactured system (FRI61F50, Thorlabs) made to mount onto a standard upright microscope. The source element is a set of five concentric fibre rings, where each ring is composed of the ends of a set of single-mode fibres that are angled toward the centre of the ring to emit light toward the sample. The maximum outer diameter of the rings is $55 \mathrm{~mm}$; each of the five rings is $70 \mu \mathrm{m}$ in width and radially spaced by $30 \mu \mathrm{m}$. The set of fibres are combined into a fibre bundle, which is coupled to a light source, offering a high degree of flexibility. Here, we couple the bundle to a halogen bulb (OSL2 Fibre Light Source; Thorlabs, USA). The geometry was modelled in the software according to the manufacturer specifications [20]. The emitting area consists of approximately ten thousand single-mode fibres. Each fibre is oriented at an angle of $10^{\circ}$ toward the centre of the ring to produce a directional lighting effect. Due to the random nature of the fibre distribution, the emitting area is effectively 

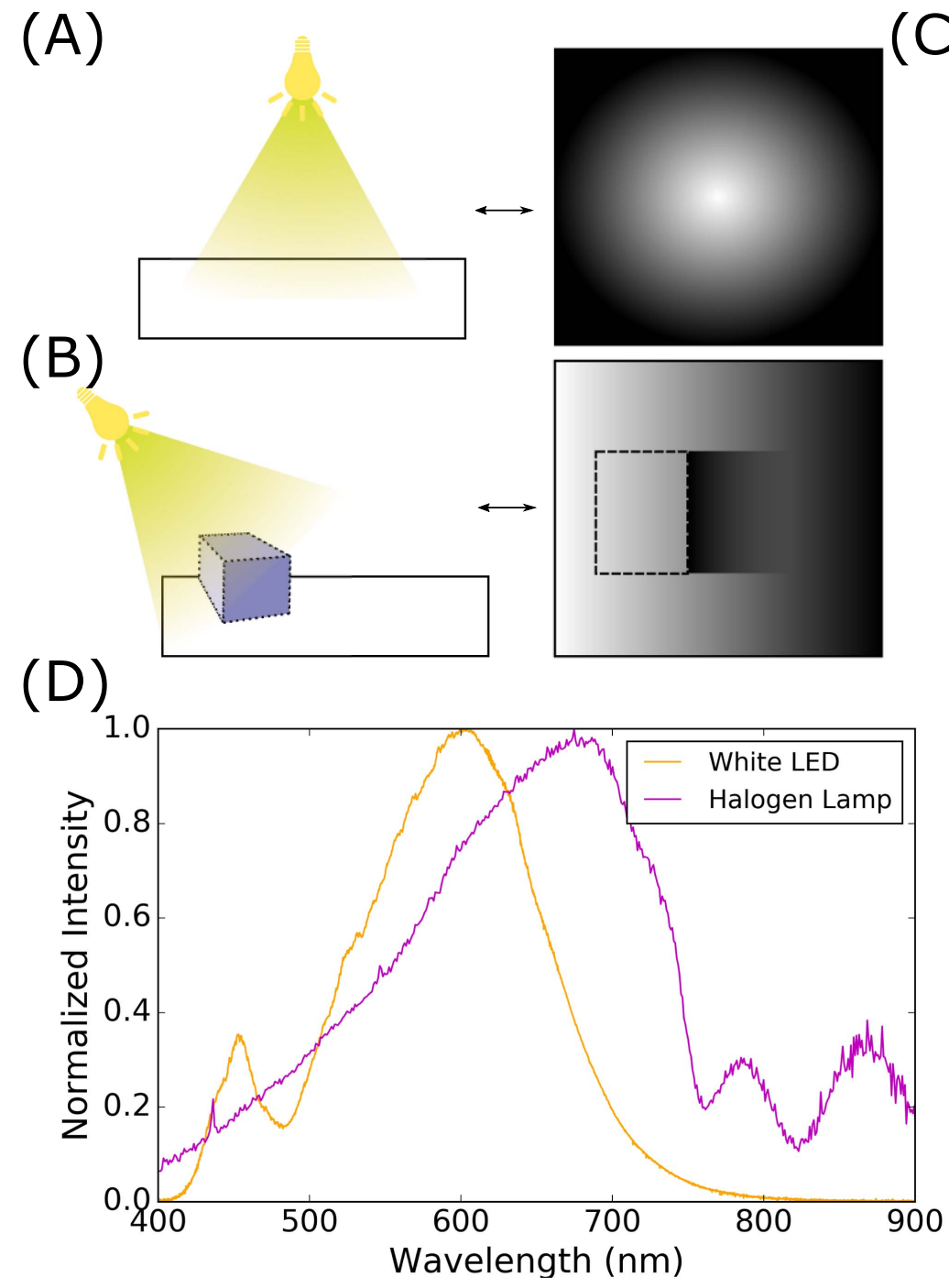

(C) $400 \mathrm{~nm}-500 \mathrm{~nm}$

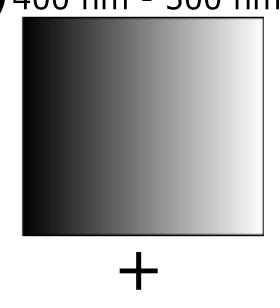

$500 \mathrm{~nm}-600 \mathrm{~nm}$

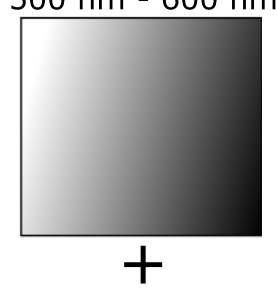

$600 \mathrm{~nm}-700 \mathrm{~nm}$

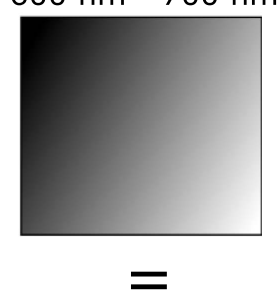

$400 \mathrm{~nm}-700 \mathrm{~nm}$

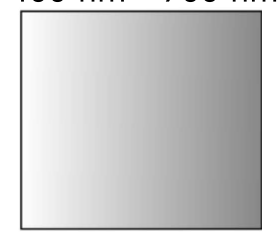

Figure 2. Different lighting conditions leading to non-uniformities. (A) Spatial non-uniformity can lead to bright and dark regions in the target plane. (B) Angular non-uniformity can cause objects to cast shadows. (C) Spectral non-uniformities can exist in small wavelength regions but not manifest in the total irradiance. (D) Spectral uniformity also occurs as a variation in the spectral power density.

approximated as a spatially uniform source, with the angular distribution matching that emitted by a single fiber in the bundle [21]. Finally, the halogen light source spectrum provided by the manufacturer was digitised into the software (figure 2(D)).

2.1.2. LED ring. The LED ring is a custom laboratory system developed for fluorescence imaging, composed of a copper ring suspended above the sample. Two colours of high power LED sources are distributed around the circumference of the ring to provide fluorescence excitation (Rebel Amber $590 \mathrm{~nm}$, Deep Red $655 \mathrm{~nm}$; Lumileds, USA). Six sources of each colour are equally spaced around the ring, with a sheet of transmissive photographic diffuser paper (Lee White Diffusion; Calumet Photographic, UK) inserted beneath the ring to homogenise the illumination [15]. We later exchange the colour LEDs for a white LED of similar optical properties to examine the spectral uniformity (figure 2(D), Rebel White, $4300 \mathrm{~K}$; Lumileds, USA). The ring geometry of the system was input into the software and the LED source elements were modelled using the geometric and emittance (spatial and angular) properties of each LED obtained from the manufacturer. The diffusing sheet was modelled as a transmissive scattering medium with Lambertian properties [22, 23].

2.1.3. Illumination dome. The illumination dome is a professionally manufactured system (MB-DL306, Vision Light Tech), composed of a hemisphere with eight emitting LED sources distributed around the inner base of the dome and an aperture located at the apex to enable imaging of samples within the dome. The inner surface of the dome is coated with a highly reflective, diffusing material to homogenise the light reaching the sample, which is placed at the centre of the hemisphere. A 4 inch $(101.6 \mathrm{~mm})$ dome diameter was selected for the purpose of this model and a 98\% reflectance coating with a Lambertian scatter profile was applied per the manufacturer specifications [24]. The same geometric and emittance properties used for the LED sources in the LED ring model are employed here. The imaging 


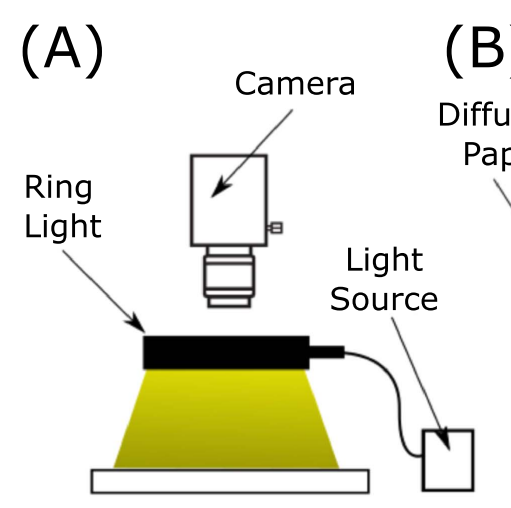

(B)

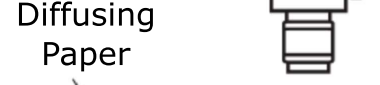

(D)

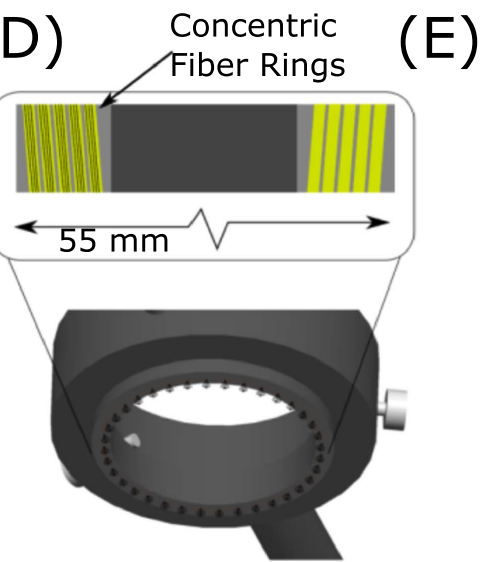

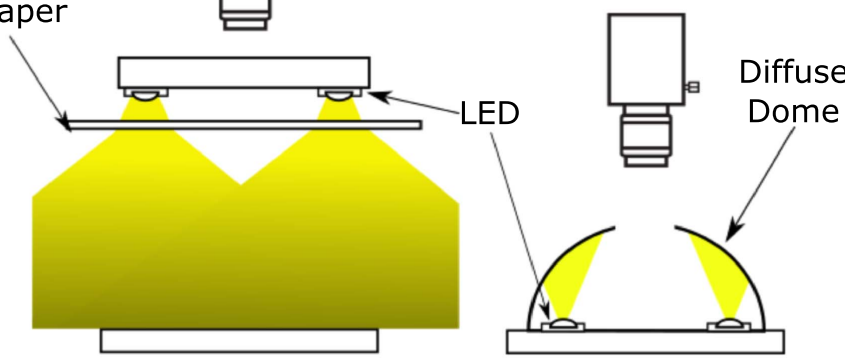
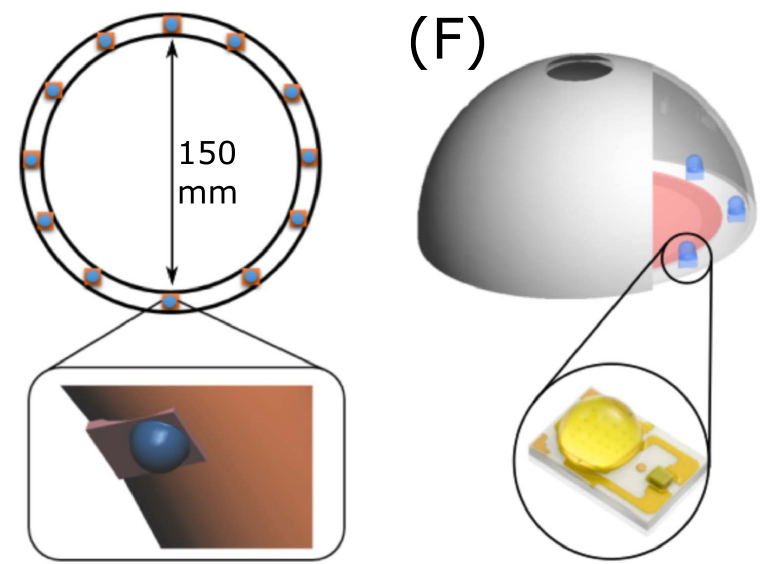

Figure 3. Schematic illustrations and solid models of the fibre ring light (A) and (D), the LED ring (B) and (E), and the illumination dome (C) and $(\mathrm{F})$ systems.

aperture was set to a radius of 0.75 inch $(19.05 \mathrm{~mm})$ to fully accommodate the tested sample size of $35 \mathrm{~mm} \times 35 \mathrm{~mm}$.

\subsection{Simulation protocol}

2.2.1. Ray number. Following the complete modelling of the geometric and optical properties of each system, the sample was set as a $35 \mathrm{~mm} \times 35 \mathrm{~mm}$ plane, with the detection surface divided into 71 bins in $x$ and $y$. For the FRL and LED ring, the working distance was set at $140 \mathrm{~mm}$, which is the manufacturer-recommended operating distance for the FRL [20]. To simulate the illumination distributions accurately, the required ray number to reduce the error below 5\% (or two standard deviations using Gaussian statistics) was computed with the standard Rose model [25], using the formula:

$$
M_{\text {min }}=\frac{1}{\eta_{\text {ray }}} \frac{A_{o}^{\prime}}{A} \frac{k^{2}}{C^{2}},
$$

where $M_{\min }$ is the minimum number of rays to achieve a given model error, $\eta_{\text {ray }}$ is the ray transfer efficiency, $A_{o}^{\prime} / A$ is the ratio of the bin area to the total sample area, equaling the number of bins used to divide the sample area, $k$ is the $Z$-score of a standard normal distribution to achieve the desired level of uncertainty (in this case 5\%) and $C$ is the level of variation that can be discerned from the background, which was chosen as $1 \%$ to allow low-contrast features to be resolved. The ray transfer efficiency differs for each system and was found by running preliminary ray traces with approximately
10000 rays; it has been shown previously that very few rays are required to accurately sample this parameter [25]. Once the ray number is set, the ray-tracing simulations were run five times for each system and averaged. The output irradiance and intensity distributions were then analysed to evaluate the uniformity using Python.

2.2.2. Assumptions and limitations. A number of assumptions were made in the construction of the models. Firstly, it is assumed that all components and associated materials properties are homogeneous and exact. Manufacturing variation and defects are not considered. In practice, manufacturing tolerances and other sources of statistical variation may alter the result. Secondly, the scattering media in the LED ring and dome systems are assumed to scatter according to Lambert's law; in practice, there may be some deviation from this expectation, particularly at extreme scattering angles. Finally, the simulations do not account for stray light in the surrounding environment, but this tends to be experimentally mitigated and has therefore not been considered in the current model.

Inherent limitations are imposed due to the nature of discretisation and computer precision. Due to the division of the detection surface into a finite number of bins, the discernible high-frequency spatial variation in the signal is theoretically limited by the Nyquist frequency. Increasing the bin number would mitigate this effect, however, computation 


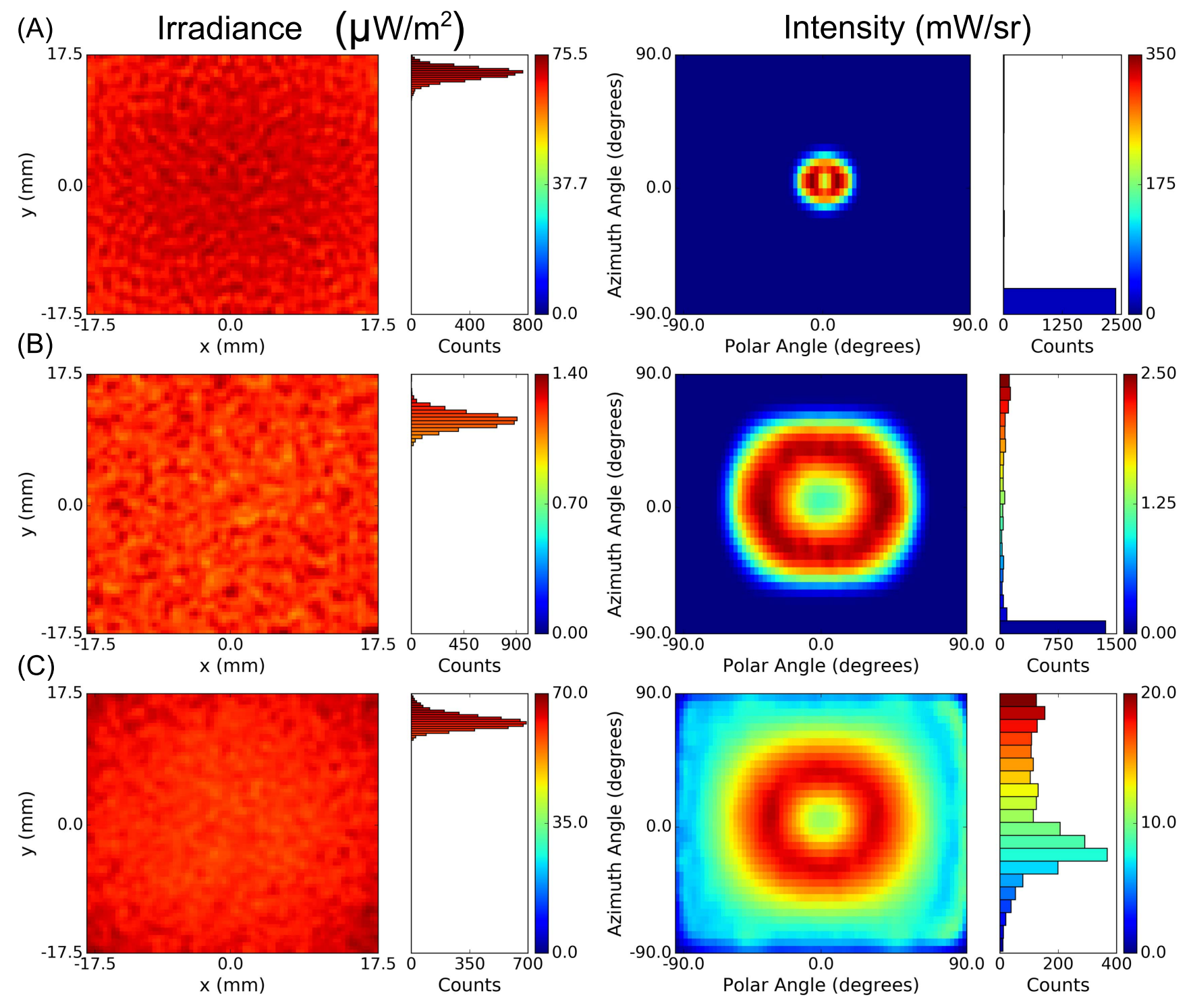

Figure 4. Illustration of spatial irradiance (left column) and angular intensity (right column) uniformity simulations for the FRL (A), LED ring (B), and illumination dome (C).

time increases with the number of bins hence these two factors must be balanced.

\subsection{Performance metrics}

To evaluate each system quantitatively, figures of merit were established to assess the performance in terms of the criteria of spatial, angular and spectral uniformity, as well as optical efficiency. For the spatial and angular uniformity results, an analysis of variance (ANOVA) test was then applied to evaluate the statistical significance of the findings.

2.3.1. Spatial uniformity. The performance metrics for spatial uniformity are computed by evaluating the irradiance distribution at the object plane. Two particular features of the distribution are of interest: the maximum deviation of illumination levels and the spread of the distribution. The former characterises the contrast by considering the extreme values in the distribution, whereas the latter provides a more statistically stable measurement to capture noise characteristics. The maximum deviation is quantified by the contrast ratio, defined as:

$$
\text { Contrast Ratio }=\frac{\max (E)-\min (E)}{\max (E)+\min (E)},
$$

where $E$ corresponds to the spatial irradiance distribution at the sample plane, in units of $\mathrm{W} \mathrm{mm}^{-2}$. This metric ranges between zero and one, where zero represents a perfectly uniform distribution. The average deviation, also known as the coefficient of variation, is used to characterise the spread of the distribution, defined as:

$$
\text { Average Deviation }=\frac{\sigma_{E}}{\bar{E}},
$$

where $\sigma_{E}$ corresponds to the standard deviation of the distribution and $\bar{E}$ is the mean value. Perfectly uniform 
(A)

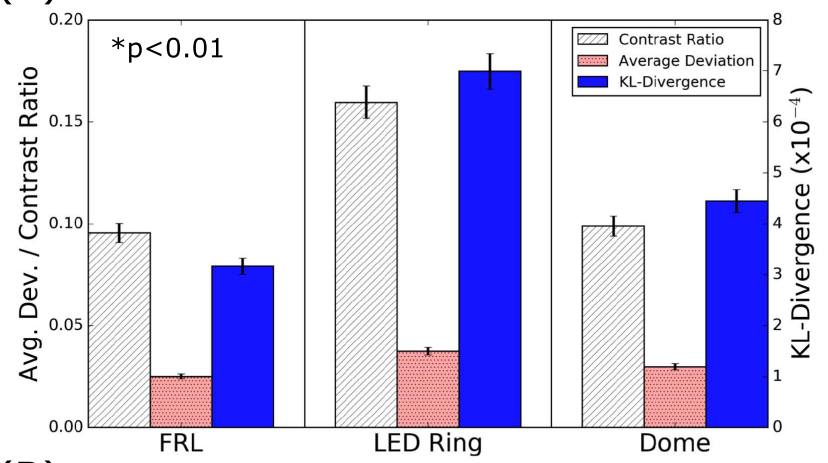

(B)

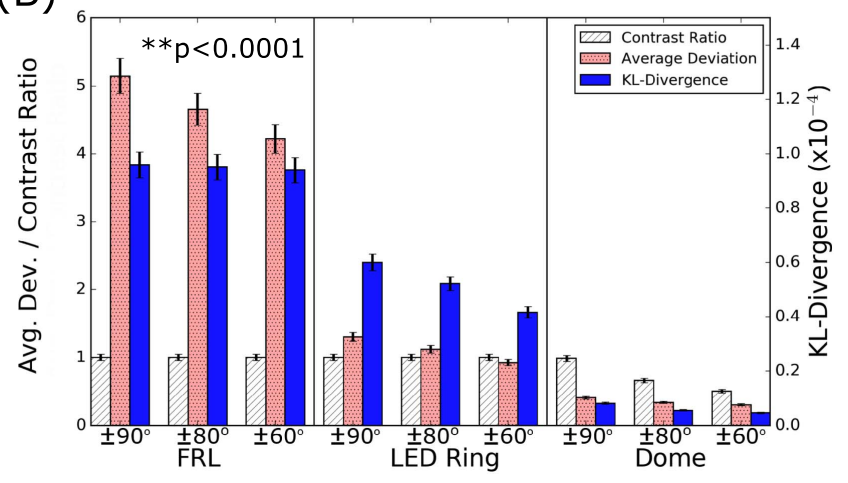

Figure 5. System performance comparison in terms of spatial (A) and angular (B) uniformity. While the FRL yields the best performance in the spatial domain, all three systems perform reasonably well. In the angular domain, the illumination dome is far superior.

illumination is once again represented by a value of zero; however, in this case the metric can exceed a value of one.

2.3.2. Kullback-Leibler divergence. While the contrast ratio and average deviation metrics are useful to quantify the variation of a relatively flat illumination distribution, shaped distributions may be desirable for some applications and an alternative approach would be required to characterise the quality. Here we introduce the KLD as a general approach to measure illumination quality, which may be applied to arbitrarily shaped illumination distributions.

The KLD is generally used to evaluate how much information is lost when a theoretical probability distribution $Q$ is used to approximate an observed distribution $P$. Here, $Q$ is given by a two-dimensional uniform distribution, which has equal value everywhere and sums to one. $P$ is the illumination distribution that is obtained through the ray-tracing simulations of each system, normalised to sum to one.

The KLD between an observed distribution $P$, and a theoretical distribution $Q$ is defined as:

$$
D_{K L}(P \| Q)=\sum_{i} P(i) \log \frac{P(i)}{Q(i)}
$$

for all $P(i) \neq 0$ where $i$ represents a discrete index corresponding to a single pixel in the observed distribution. Wherever $P(i)=0$, the contribution from the $i$ th term is nulled, as $\lim _{x \rightarrow 0} x \log x=0$. Equation 4 can then be normalised to be bounded by zero and one through the nonlinear transformation:

$$
D=1-\exp \left(-D_{\mathrm{KL}}\right)
$$

With this normalisation, the value of $D$ ranges between zero and one, where a value of zero indicates a perfectly uniform distribution (consistent with the metrics defined in section 2.3.1).

2.3.3. Angular uniformity. The angular uniformity of a system is characterised by evaluating the intensity distribution of a system in units of $\mathrm{W}$ steradian $^{-1}$. The metrics defined above are used with one additional qualification imposed. In our geometry, the sample is limited to receiving light from a hemispherical solid angle, as the rear side of the object is placed on a flat surface, such as a laboratory bench. Furthermore, the system geometry of the FRL and LED ring further limits this angular subtense of incident light. We therefore calculated the angular uniformity metrics for the full $\pm 90^{\circ}$ hemisphere as well as for subregions of $\pm 80^{\circ}$ and $\pm 60^{\circ}$.

2.3.4. Spectral uniformity. In the spectral domain, uniformity can be defined in two distinct ways. The first consideration is the shape of the illumination spectrum. While a flat spectrum is highly desirable to have equal spectral representation across the object, commonly used sources such as tungsten filaments, halogen bulbs, [26, 27] or white light LEDs [16] have distinctly non-flat distributions. To improve this form of spectral uniformity, filters can be employed to selectively attenuate the source power and, more recently, custom LED light sources are being tailored to produce a tunable spectrum [28]. We do not quantitatively measure this non-uniformity, as we are focused on evaluating the optical processes involved in distributing the light.

The second aspect of spectral uniformity is the wavelength dependence of spatial and angular uniformity. The LED ring and illumination dome were first altered to produce broadband illumination by replacing the narrowband LED sources with white-light LEDs (Rebel White, $4300 \mathrm{~K}$; Lumileds, USA) with similar emission properties to the original sources. The spectral uniformity was then assessed by re-calculating all metrics in narrow wavelength bandpasses of $100 \mathrm{~nm}$ throughout the full illumination spectrum, which is set to range between 400 and $1000 \mathrm{~nm}$ to represent visible and near-infra-red imaging. A linear regression is applied to each set of measurements and by testing a null hypothesis that the linear fit possesses zero gradient, it was determined whether any significant trend exists. We applied a relatively coarse 'multispectral' rather than hyperspectral wavelength sampling in this work due to the computational expense of the calculation for each band. While this may extrapolate to the hyperspectral case for optical elements whose spectral response varies slowly as a function of wavelength, such as the scattering elements employed here, for optical elements 


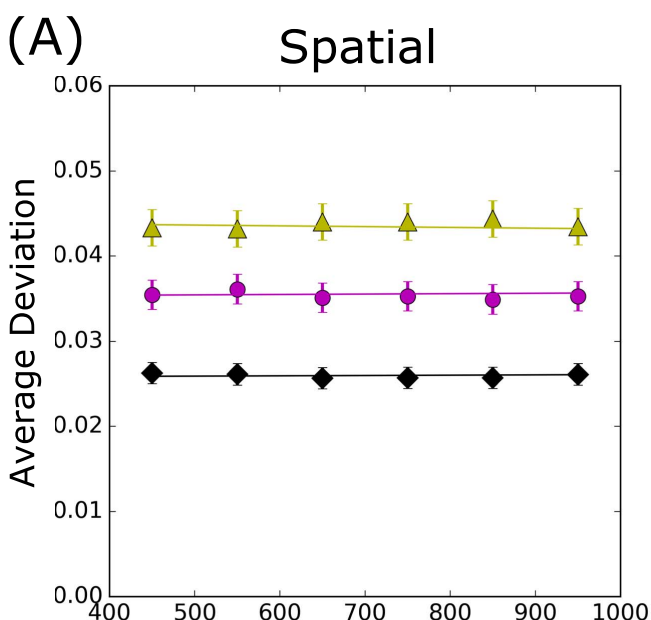

(C)

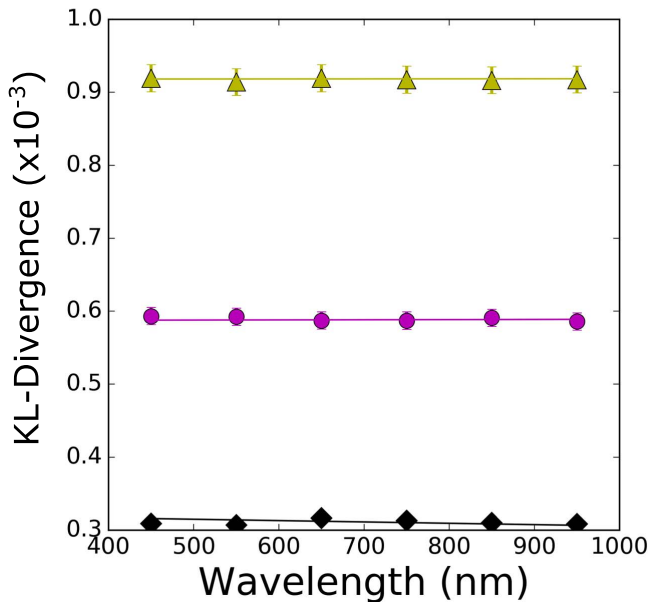

(B)

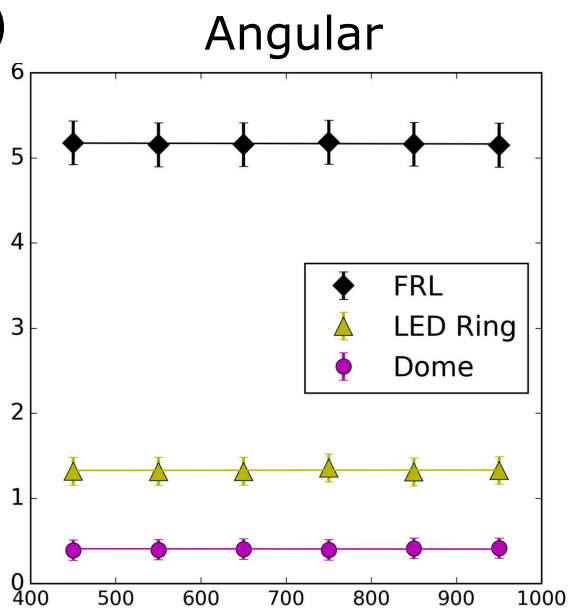

(D)

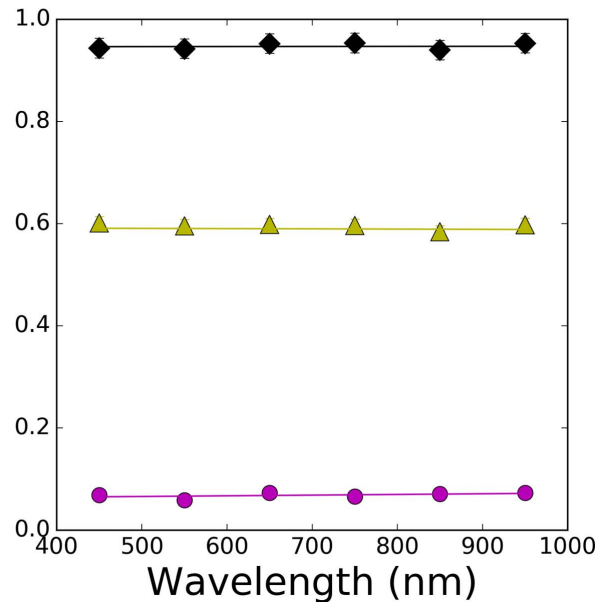

Figure 6. Spectral average deviation (top row) and KLD (bottom row) for spatial (A), (C) and angular (B), (D) uniformity.

Table 1. Results of the hypothesis test ( $p$-values) to determine if the performance metrics possess any linear trend across the wavelength range of $400-1000 \mathrm{~nm}$. A high $p$-value indicates high confidence that the metric has no discernible dependence on wavelength.

\begin{tabular}{lccccc}
\hline & \multicolumn{2}{c}{ Average deviation } & & \multicolumn{2}{c}{ KL-divergence } \\
\cline { 2 - 3 } \cline { 6 - 6 } System & Spatial & Angular & & Spatial & Angular \\
\hline FRL & 0.829 & 0.257 & & 0.409 & 0.467 \\
LED ring & 0.589 & 0.812 & & 0.393 & 0.897 \\
Illumination dome & 0.524 & 0.412 & & 0.756 & 0.438 \\
\hline
\end{tabular}

with high frequency spectral variation, finer sampling would be necessary to truly represent the HSI case.

2.3.5. Efficiency. System efficiency is an important parameter, as it provides insight into operational characteristics such as power use, heat dissipation, test duration, etc. As such, the efficiency for each system is characterised using the equation:

$$
\eta=\frac{\mathrm{P}_{\text {sample }}}{P_{\text {emitted }}} \eta_{\mathrm{c}}
$$

where $P_{\text {emitted }}$ corresponds to the power leaving the emitting area of the source, $P_{\text {sample }}$ represents the total power received by the $35 \mathrm{~mm} \times 35 \mathrm{~mm}$ sample plane and $\eta_{\mathrm{c}}$ refers to the system coupling efficiency, which accounts for losses in the system prior to the emission of light. For the simulations in this study, $P_{\text {emitted }}$ was normalised to $1.0 \mathrm{~W}$; therefore the power ratio term in the expression is simply found by integrating the total power over the detection surface.

\subsubsection{Spatial uniformity dependence on working distance.}

The FRL and LED ring systems have variable working distances, defined as the distance between the source and the sample, which will impact illumination uniformity. For the dome, this distance is fixed and therefore the dome is not considered. In particular, the spatial distribution can evolve significantly over a small distance depending on the incident ray angle. To evaluate the dependence on the working distance, a series of simulations were run for both the FRL and LED ring systems, where the distance between the source and object was varied between 30 and $140 \mathrm{~mm}$ in increments of $10 \mathrm{~mm}$. For the LED ring system, the distance was measured between the object and the diffuser paper, resulting in an overall shift of the working distance of $40 \mathrm{~mm}$ compared to the FRL. Here, only the spatial uniformity is interrogated; the ray angle of incidence is not affected as working distance 
(A)

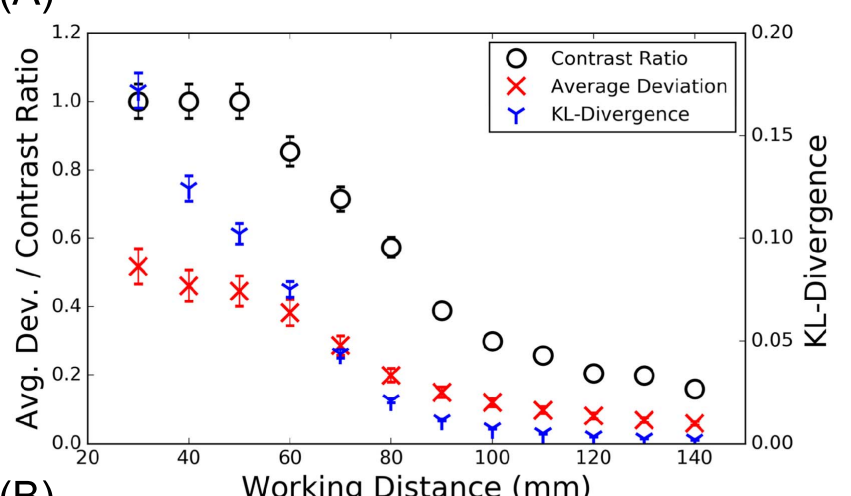

(B)

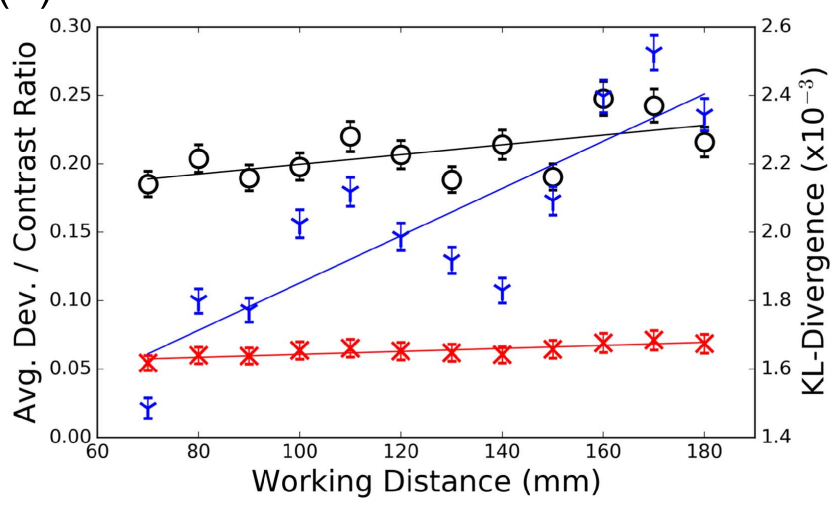

Figure 7. Spatial uniformity as a function of working distance for the FRL (A) and the LED ring (B). The FRL shows a large dependence on the working distance, while the LED ring exhibits only a slight trend. The error bars for each point represent the uncertainty for each simulated value.

is changed, implying that the angular distribution remains approximately constant. It could potentially be affected by vignetting but this indirect effect is not considered here.

\section{Results}

\subsection{Spatial and angular uniformity}

The spatial and angular distributions simulated for the FRL (figure 4(A)), LED ring (figure 4(B)) and illumination dome (figure $4(\mathrm{C})$ ) were used to compute the average deviation, contrast ratio, and KLD (figure 5). Qualitatively, while all three systems perform well in the spatial domain, the angular distributions are highly non-uniform.

Quantification of the uniformity metrics (contrast ratio, average deviation and KLD) shows that the FRL yields the lowest values, i.e. highest spatial uniformity, in all cases ( $p<0.01$ by ANOVA). The FRL is closely followed by the dome (figure 5(A)). In terms of angular uniformity, the FRL yields the poorest performance by an order of magnitude (figure 5(B)), with the LED ring only slightly better. This is expected given that the geometry of the ring systems fundamentally limit the angle at which light can strike the object. The directional nature of fibre illumination in the FRL results in a further restricted angular distribution. Assessment of angular uniformity highlights the limitations of the contrast ratio metric; the contrast ratio is equal for both the FRL and LED ring, but the LED ring is clearly more uniform by visual inspection (figure 4(B)) and according to the average deviation and KLD. Angular uniformity is optimal using the illumination dome ( $p<0.0001$ by ANOVA).

\subsection{Spectral uniformity}

All three systems perform consistently across the spectrum in both the spatial and angular domains (figure 6). We applied a linear regression analysis to determine whether the linear fit possesses zero gradient; in all cases we found $p>0.25$ (table 1) indicating that no significant trends exist in the data. These results support the hypothesis that the coarse spectral sampling of $100 \mathrm{~nm}$ is representative of the spectral behaviour for optical elements with relatively flat spectral responses such as diffusers; any deviations, whether with a functional form or random, would be reflected in the form of a lower $p$ value in the hypothesis test.

\subsection{Efficiency}

To assess efficiency, we scaled the source power to $1.0 \mathrm{~W}$ and integrated the incident power over the object plane to yield the total power reaching the surface, accounting for any coupling losses not captured by the models. The efficiency of the illumination dome was highest at $7.57 \% \pm 0.51 \%$, with the FRL second at $4.54 \% \pm 0.33 \%$ and the LED ring poorest at $0.15 \% \pm 0.15 \%$.

\subsection{Spatial uniformity dependence on working distance}

Analysing the spatial uniformity as a function of working distance indicates that the FRL has a significant increase in uniformity as the working distance is increased from $30 \mathrm{~mm}$ to approximately $120 \mathrm{~mm}$ (figure 7(A)), but beyond $120 \mathrm{~mm}$, changing the working distance has little effect. The LED ring exhibits comparatively little variation (figure 7(B)), though it exhibits a non-zero linear trend for all three metrics (contrast: $p<0.05$; average deviation and KLD: $p<0.001$ ), indicating decreased uniformity at greater working distances.

\section{Discussion}

Illumination uniformity is crucial for accurate analysis in HSI. Here, we described the modelling and evaluation of three illumination systems commonly used in wide-field HSI. Using two optical design and analysis software packages, the geometric and optical properties were modelled and simulations were run to determine the irradiance and intensity distributions incident on the sample. Spatial, angular, and spectral uniformity were assessed, along with the efficiency, using two common metrics (contrast ratio and average deviation) and a new approach based on the KLD. The KLD produces a relative value consistent with the average deviation but is compatible with with a range of other applications including: beam shaping [29] and profiling [30]; modal noise characterisation $[31,32]$; as well as structured illumination 
and projection [33-35]. The KLD therefore presents a broadly applicable metric with great potential for the general assessment of illumination quality.

All three systems yield high spatial and spectral uniformity, with the FRL performing the best spatially albeit by a narrow margin. The illumination dome geometry performs significantly better than the FRL or LED ring in terms of angular uniformity and efficiency, which taken together with the favourable spatial and spectral uniformity results, indicate that this geometry is most suitable for biomedical HSI.

While these results are encouraging, we identified a number of challenges for future investigation. For spectral uniformity, we used a relatively wide bandpass of $100 \mathrm{~nm}$. In applications such as fluorescence imaging, where potential non-uniformities may occur on narrower ranges, such as $10 \mathrm{~nm}$ or less, simulating illumination on this scale will be necessary but computationally expensive. All three systems performed relatively poorly in the angular domain compared to the respective spatial distributions. This result suggests that designing new approaches that improve on this aspect of illumination uniformity would be of value for implementation of future HSI systems. For low light level applications such as fluorescence imaging, current illumination systems may not be sufficient to maximise detection capabilities. The modelling approach demonstrated here could in future be used to rapidly evaluate novel illumination designs that improve upon angular uniformity or maximise performance by multiplexing broadband and narrowband illumination.

\section{Conclusion}

We simulated the illumination distributions of three source geometries commonly used in wide-field HSI. We compared three metrics to assess their performance: contrast ratio; average deviation; and the KLD. The average deviation and KLD prove to be equivalently suitable for this analysis; taken together with the broad potential of the KLD for illumination quality our findings suggest that this metric is the most appropriate. The system analysis results indicate that an illumination dome provides the best angular uniformity along with high efficiency, whereas the fibre ring light yields the most uniform spatial illumination. Tailoring systems to satisfy the unique illumination requirements for a given application may therefore be necessary to maximize the potential of HSI systems in wide-field biomedical imaging applications.

\section{Acknowledgments}

We would like to thank Dr James Joseph and Dr Jonghee Yoon for manuscript comments and Dr John Koshel of the University of Arizona for assistance with the optical design and analysis software, as well as theoretical support provided in the Illumination Engineering course at the University of Arizona. TWS is funded by the Winston Churchill Foundation of the United States. ASL is funded by the EPSRC, the George and Lillian Schiff Foundation and the Foundation
Blanceflor. SEB is funded by CRUK (C14303/A17197, C47594/A16267 and C47594/A21102) and the European Union's Seventh Framework Programme (FP7/2007-2013) under grant agreement number FP7-PEOPLE-2013-CIG630729. We also acknowledge support from a University of Cambridge MRC Confidence in Concept Award.

\section{References}

[1] Lim H and Murukeshan V 2015 Pushbroom hyperspectral imaging system with selectable region of interest for medical imaging J. Biomed. Opt 20046010

[2] Lu G and Fei B 2014 Medical hyperspectral imaging: a review J. Biomed. Opt. 1910901

[3] Grusche S 2014 Basic slit spectroscope reveals threedimensional scenes through diagonal slices of hyperspectral cubes Appl. Opt. 53 4594-603

[4] Kester R T, Bedard N, Gao L and Tkaczyk T S 2011 Real-time snapshot hyperspectral imaging endoscope J. Biomed. Opt. 16056005

[5] Katrašnik J, Pernuš F, Likar B, Górski K, Hivon E, Banday A, Wandelt B, Hansen F, Reinecke M and Bartelmann M 2013 A method for characterizing illumination systems for hyperspectral imaging Opt. Express 21 209-17

[6] Tack N, Lambrechts A, Soussan P and Haspeslagh L 2012 A compact, high-speed, and low-cost hyperspectral imager Proc. SPIE $82668266 \mathrm{Q}$

[7] Plaza A et al 2009 Recent advances in techniques for hyperspectral image processing Remote Sens. Env. 113 (Suppl.) 1

[8] Orfanoudaki I M, Themelis G C, Sifakis S K, Fragouli D H, Panayiotides J G, Vazgiouraki E M and Koumantakis E E 2005 A clinical study of optical biopsy of the uterine cervix using a multispectral imaging system Gynecol. Oncol. 96 $119-31$

[9] Balas C 2001 A novel optical imaging method for the early detection, quantitative grading, and mapping of cancerous and precancerous lesions of cervix IEEE Trans. Biom. Imag. 48 96-104

[10] Luthman A S, Dumiru S, Quirós-Gonzalez I and Bohndiek S E 2016 Hyperspectral fluorescence imaging with multiwavelength LED excitation SPIE BiOS pp 971110-1

[11] Waterhouse D J, Joseph J, Neves A A, di Pietro M, Brindle K M, Fitzgerald R C and Bohndiek S E 2016 Design and validation of a near-infrared fluorescence endoscope for detection of early esophageal malignancy J. Biomed. Opt. 21 84001

[12] Polder G W, van der Heijden G W A M and Young I T 2002 Spectral image analysis for measuring ripeness of tomatoes Trans. ASAE 45 1155-61

[13] Sun D 2010 Hyperspectral Imaging for Food Quality Analysis and Control (Amsterdam: Elsevier)

[14] Janesick J R 2007 Photon Transfer (Bellingham, WA: SPIE Press)

[15] Luthman A S and Bohndiek S E 2015 Experimental evaluation of a hyperspectral imager for near-infrared fluorescent contrast agent studies SPIE BiOS p $93180 \mathrm{H}$

[16] Kerr P H, Fisher E M and Buffington M L 2008 Dome Lighting for insect imaging under a microscope $A m$. Entomol. 54 198-200

[17] Kawada R and Buffington M L 2016 A scalable and modular dome illumination system for scientific microphotography on a budget PLOS ONE 115

[18] Moreno I 2010 Illumination uniformity assessment based on human vision Opt. Lett. 35 4030-2 
[19] Anbarjafari G 2015 An objective no-reference measure of illumination assessment Meas. Sci. Rev. 15 319-22

[20] Thorlabs 2015 Fiber ring illuminator for OSL2 fiber light source www.thorlabs.com/thorproduct.cfm? partnumber $=$ FRI61F50

[21] Nemoto S 1984 Illuminance distribution produced by a fiber bundle Appl. Opt. 23 2859-60

[22] Adams A, Fahrenwald M and Do L 2007 Measurement and modeling of light transmission through turbid media $J$. Ark. Acad. Sci. $612-4$

[23] Borch J, Lyne M B, Mark R E and Habeger C C Jr 2002 Handbook of the Physical Testing of Paper (Boca Raton, FL: CRC Press)

[24] Vision Light Tech 2015 MB-DL306 Datasheet www. visionlighttech.com/sap/datasheets/MB-DL306-xxx.pdf

[25] Koshel R J 2004 Illumination Engineering (New York: Wiley)

[26] Kim M S, Chen Y and Mehl P M 2001 Hyperspectral reflectance and fluorescence imaging system for food quality and safety Amer. Soc. Agr. Eng 44 721-9

[27] Huang H, Liu L and Ngadi M O 2014 Recent developments in hyperspectral imaging for assessment of food quality and safety Sensors 14 7248-76
[28] Speier I and Salsbury M 2006 Color temperature tunable white light LED system 6th Int. Conf. on Solid State Lighting vol 6337, p 63371F

[29] Xie C, Gupta R and Metcalf H 1993 Beam profile flattener for Gaussian beams Opt. Lett. 18 173-5

[30] Dickey F M and Holswade S C 1996 Gaussian laser beam profile shaping Opt. Eng. 35 3285-95

[31] Kanada T and Aoyama K 1983 Modal noise evaluation in multimode fiber transmission Opt. Lett. 8 339-41

[32] Mourka A, Mazilu M, Wright E M and Dholakia K 2013 Modal characterization using principle component analysis: application to Bessel, higher-order Gaussian beams and their superposition Sci. Rep. 31422

[33] Krzewina L G and Kim M K 2007 Structured illumination imaging Microsc. Image Process. 469-97

[34] Cuccia D J, Bevilacqua F, Durkin A J and Tromberg B J 2005 Modulated imaging: quantitative analysis and tomography of turbid media in the spatial-frequency domain Opt. Lett. 30 1354-6

[35] Stallinga S, Chakrova N and Rieger B 2015 Studying different illumination patterns for resolution improvement in fluorescence microscopy Opt. Express 23 24692-701 